GEISSMAN, T. A., AND LISCHNER, H. 1952. Flavanones and related compounds. VII. The formation of 4, 6, 3', 4'-tetrahydroxy-2-benzylcoumaranone-3 by the sodium hydrosulphite reduction of quercetin. J. Am. Chem. Soc., 74, 3001-3004.

GILbert, R. I. 1971. An unusual anthocyanin in Antirrhinum majus. Phytochem., 10, 2848-2849. gIlbert, R. I. 1972. Pelargonidin-3-glucoside in Antirrhinum majus. Phytochem., 11, 2360. GILbERT, R. I. 1973. Chalcone glycosides of Antirrhinum majus. Phytochem., 12, 809-810.

GRISEBACH, H. 1972. Enzymology and regulation of flavonoid biosynthesis in plants and plant cell cultures. Phytochem., 11, 862-863.

HARBORNE, J. B. 1963. Plant polyphenols. X. Flavone and aurone glycosides of Antirrhinum. Phytochem., 2, 327-334.

HARborne, J. B. 1967. Comparative biochemistry of the flavonoids. Academic Press.

HARRISON, B. J., AND FINGHAM, J. R. s. 1964. Instability at the pal locus in Antirrhinum majus. I. Effects of environment on frequencies of somatic and germinal mutation. Heredity, $19,237-258$.

SCOTT-MONCRIEFF, R. 1937. Biochemistry of flower colour variation. In Perspectives in Biochemistry, Ed. J. Needham and D. E. Green, 230-243. Cambridge University Press. STICKLAND, R. G., AND HARRISON, B. J. 1973. Precursors and pigmentation in Antirrhinum majus. John Innes Annual Report.

\title{
PRECURSORS AND GENETIC CONTROL OF PIGMENTATION
}

\section{GENOTYPE ANALYSIS OF PIGMENT CONTROLLING GENES IN} ACYANIC PHENOTYPES IN ANTIRRHINUM MAJUS

\author{
B. J. HARRISON and R. G. STICKLAND \\ John Innes institute, Colney Lane, Norwich NOR 7OF
}

Received 19.ii.74

\section{Summary}

Imbibition of dihydroquercetin, dihydrokaempferol and naringenin by the flowers of certain genotypes of Antirrhinum majus enables rapid identification of hypostatic genes involved in pigment synthesis. Induced synthesis of anthocyanidin in acyanic flowers has been shown to occur after imbibition of particular acyanic flower homogenates.

\section{InTRODUCtION}

THE pigment pathway in Antirrhinum majus culminating in anthocyanidin can be blocked in several places by known genes. It has been found possible to synthesise anthocyanidins in acyanic flowers by administering the flavanonol precursors dihydroquercetin, dihydrokaempferol and dihydromyricetin (Stickland and Harrison, 1974). The presence of homozygous nivea (niv) in the genotype precludes the identification by visual inspection of the hypostatic genes incolorata (inc), delila (del), pallida ( pal) alleles and eosinea (eos) except by breeding techniques; however, the use of these precursors and the flavanone, naringenin, has enabled many genotypes containing these genes to be readily identified.

\section{Materials AND methods}

With the exception of naringenin (5,7,4'-trihydroxy flavanone) obtained from Koch-Light Laboratories Ltd. and used as a saturated aqueous solution all other materials and methods are as described by Stickland and Harrison (1974). The homogenates were prepared from macerated and ground 


\section{Plate I}

Left. Untreated corolla of homozygous incolorata:pallida-recurrens.

Right. Similar corolla treated with dihydroquercetin. The mutant sites of pallida-recurrens $\rightarrow$ Pallida are indicated by the flakes of synthesised cyanidin. 


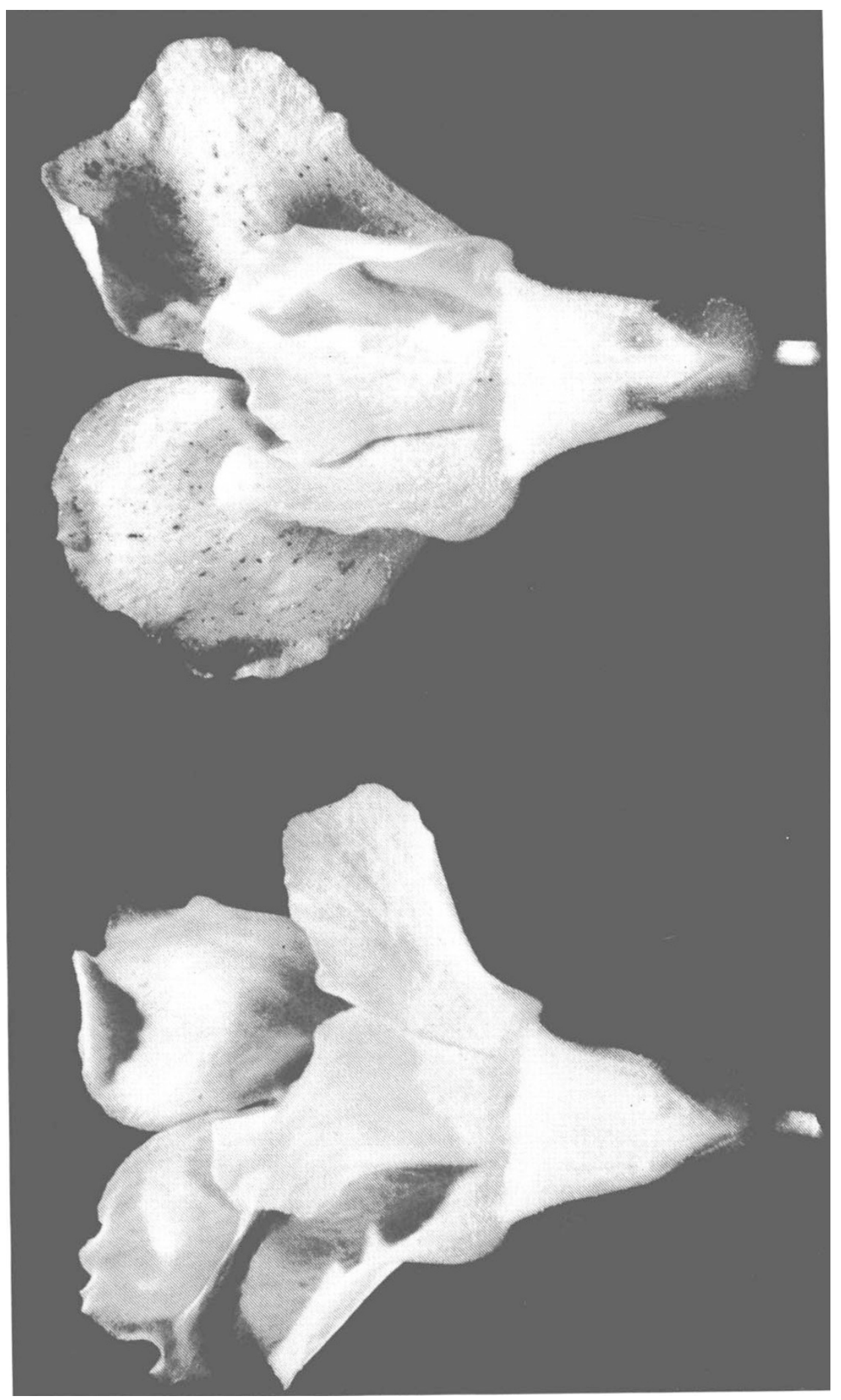


flower material to which approximately 10 per cent distilled water was added. The unfractionated homogenate was administered to the cut flowers by immersion of the corolla tube in a similar way to that adopted for chemical imbibition.

\section{Results AND GONGlusions}

The genotype niv:inc (or Inc):Pal is phenotypically niv; the imbibition of dihydroquercetin (DHQ) initiates cyanidin synthesis (fig. 1, 1) and this

Phenotype Genotype and added precursor Result $\begin{gathered}\text { Gene } \\ \text { deduction }\end{gathered}$

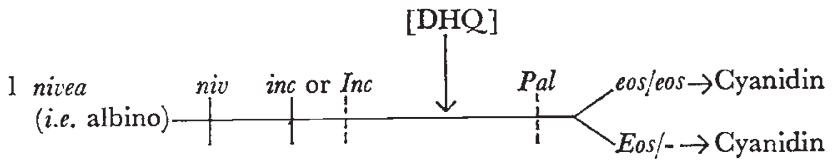

Pallida

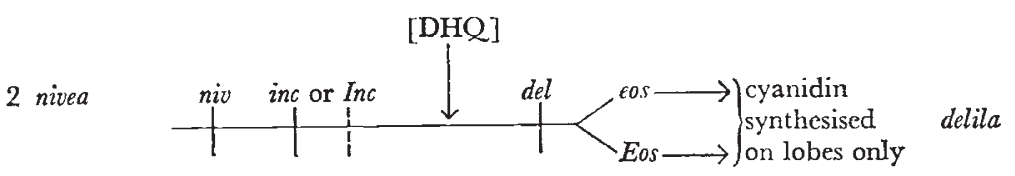

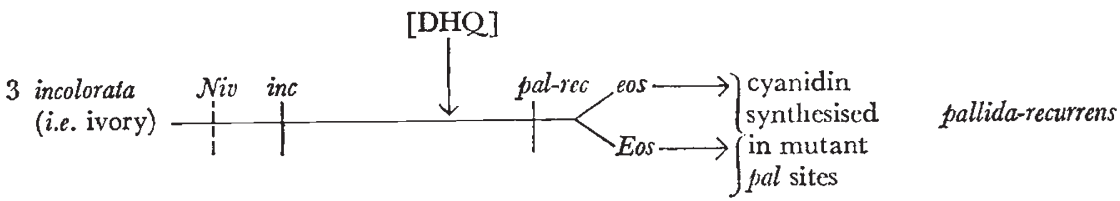

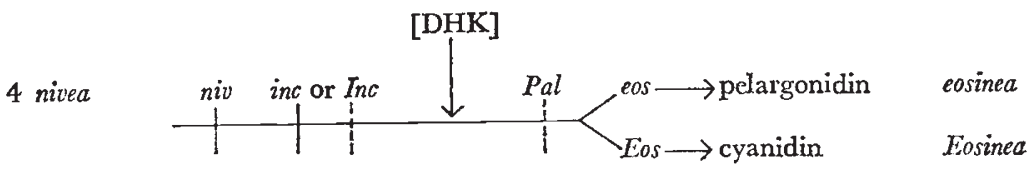

5 nivea

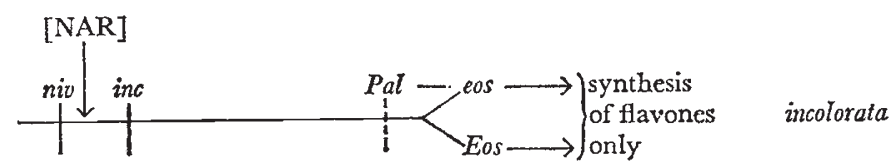

6 nivea

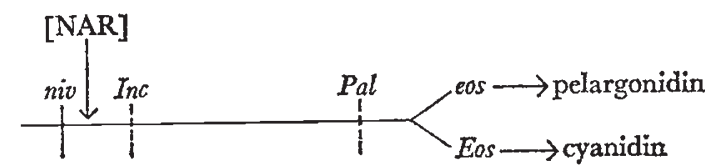

Incolorata:Pallida:
eosinea
Incolorata:Pallida: Incolorata:Pallida:
Eosinea

FIG. 1.-Abbreviations: [DHK], [DHQ] and [NAR], point of administration of dihydrokaempferol, dihydroquercetin or naringenin respectively. Gene names are given in full in the last column. Genetic blocks and functional genes are indicated by solid and broken upright bars respectively.

$33 / 1-\mathrm{H}$ 
synthesis occurs in both inc and Inc plants provided that the plant is Pal. It is, therefore, possible to deduce the presence of the dominant $\mathrm{Pal}$ component. The DHQ precursor initiates cyanidin only, irrespective of whether the plant is eos (gene for pelargonidin) or Eos (gene for cyanidin). The use of the precursor dihydrokaempferol (DHK) enables the identification of the eos/eos genotype since flowers bearing the Eos allele can add a 3-hydroxyl group to form cyanidin (fig. 1, 4).

Homozygous del flowers have no anthocyanidin in the corrola tube. The imbibition of DHQ in an acyanic genotype that is also del causes synthesis of anthocyanidin only in the lobes, the tube remaining acyanic (Fig. 1, 2).

Some pallida alleles can simulate the effect of inc; for example, pal-tincta is usually acyanic and a pal-tubocolorata plant that is also del is not recognisable since the ring of pigment on the base of the corolla tube is unexpressed; a pallida-recurrens (pal-rec) plant that is not somatically mutating is also acyanic (Harrison and Fincham, 1964). The imbibition of DHQ on a flavone producing plant (i.e. $\mathcal{N i v}$ ) produces cyanidin if inc: $P$ al but no cyanidin is synthesised if the block is due to a pal allele.

The mutability of the unstable pal-rec allele has been shown to be highly sensitive to temperature during flower development (Harrison and Fincham, 1964) and to a genetic stabiliser system (Harrison and Fincham, 1968). The possibility of genetic blocks in the pigment pathway as an influence on the genetic instability was also considered. The genotype Niv:inc:pal-rec is normally unable to express mutations of pal-rec to Pal because of the acyanic block of inc. However, the imbibition of $\mathrm{DHQ}$ by such a genotype enables recognition of somatic mutations in corolla tissue (fig. 1, 3). Plate I shows the effect of DHQ on the otherwise acyanic corolla and the readily identifiable mutant areas. The incorporation of DHQ has thus conclusively shown that mutations of pal-rec can occur somatically in a plant whose pigment pathway is genetically blocked; however, it does not, at this stage, invalidate the, perhaps, remote possibility that the frequency of mutations may be affected.

The incorporation of the analogue of apigenin, naringenin, into the earlier part of the pigment pathway permits the identification of inc in a niv plant (fig. 1, 5). The inc allele only blocks the further development of flavanone to flavanonol, and synthesis of flavone, which can easily be identified chromatographically, follows the imbibition of naringenin. Naringenin, in addition to the use of $\mathrm{DHK}$, enables the recognition of the eos versus Eos constitution (fig. 1, 6) in a niv:Inc: Pal plant. Pelargonidin is synthesised in an eos/eos genotype while in an Eos/- genotype cyanidin is produced.

In addition to the chemical precursors mentioned above, natural precursors obtained from macerated and ground flowers have initiated the synthesis of anthocyanidin. A homogenate of $\mathcal{N i v}:$ Inc: Pal:eos (a pelargonidin producing plant) induces the formation of cyanidin in $\mathcal{N i v}:$ inc:Pal:Eos (an acyanic plant in which cyanidin is blocked by inc). This is comparable to the initiation of cyanidin synthesis by DHK (the precursor for pelargonidin) in this genotype. This technique enables the recognition of the constitution in respect of the eos locus of the recipient plant. A homogenate of genotype $\mathcal{N i v}$ :inc:Pal was administered to niv:Inc:Pal and a low level of anthocyanidin synthesis occurred after two days (fig. 2). As no precursor would be in the latter genotype that was not also present in the recipient 
Phenotype Genotype Result

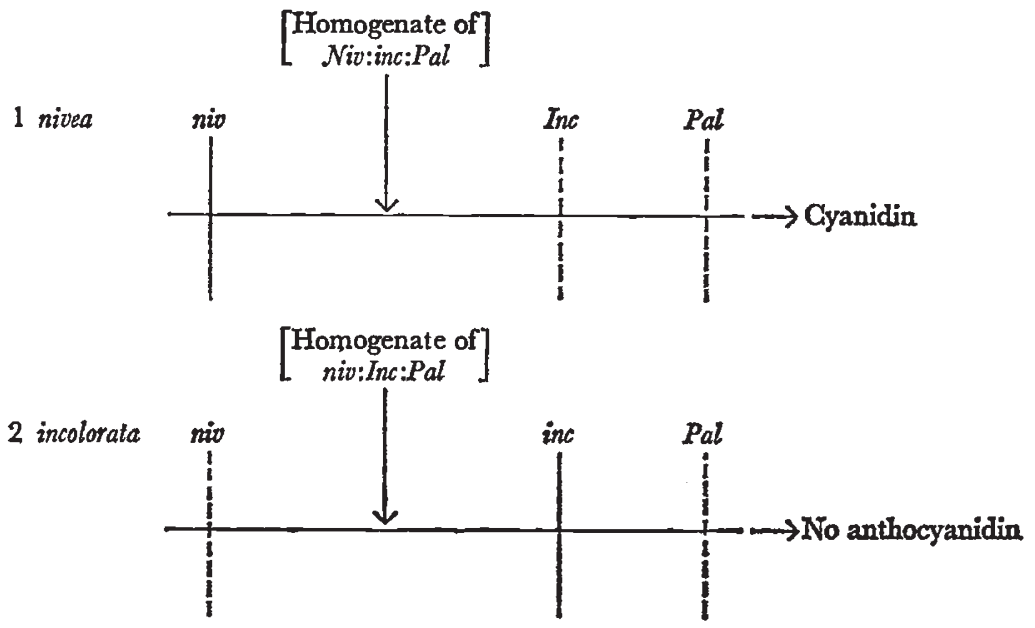

Frg. 2

the reciprocal treatment would be expected to be negative. This prediction was confirmed. Thus from two acyanic stocks of different genetic constitution a reciprocal difference in pigment release was observed from the use of homogenised flower material. It was possible from this result to deduce that the material containing the epistatic niv gene was also carrying Inc, as otherwise no anthocyanidin would be synthesisable.

\section{REFERENGES}

HARRISON, B. J., AND FINCHAM, J. R. s. 1964. Instability at the pal locus in Antirhinum majus. 1. Effects of environment on frequencies of somatic and germinal mutation. Heredity, 19, 237-258.

HARRISON, B. J., AND FINCHAM, J. R. s. 1968. Instability at the pal locus in Antirhinum majus. 3. A gene controlling mutation frequency. Heredity, 23, 67-72.

STICKLAND, R. G., AND HARRISON, B. J. 1974. Precursors and genetic control of pigmentation.

1. Induced biosynthesis of pelargonidin, cyanidin and delphinidin in Antirrhinum majus.

Heredity, 33, 108-112. 